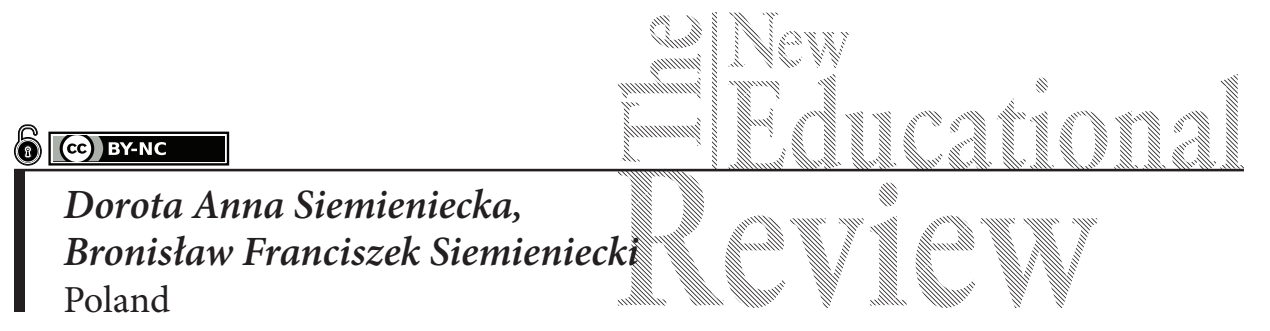

\title{
Cyberspace in the Perspective of Cognitive Pedagogy
}

DOI: 10.15804/tner.2018.54.4.13

\begin{abstract}
The article discusses the problems of human functioning in cyberspace seen from the perspective of cognitive pedagogy. The focus was on the area of communication, information processing by people and problems related to the language of the media. The understanding of the concepts of cyberspace and virtual reality from the position of cognitive pedagogy has been defined. A number of phenomena have been presented, such as: multitasking, cognitive load, sequencing mechanism, compression and addiction.
\end{abstract}

Keywords: cognitive pedagogy, cyberspace, virtual reality

Rapid development and the growing popularity of cyberspace have contributed to the increase in pedagogues' interest in the possibilities of its use in education. Initially, the focus was on the technological side of cyberspace and especially on the support of network tools. However, the need to include teaching and cognitive knowledge in the teaching process was soon discovered. The change in the visual aspect of the educational use of cyberspace is significantly influenced by extensive discussions covering the cultural and environmental foundations of social evolution. The effect of these debates were analyses made by the creators of The Third Culture, presented, among others, by John Brockman (1995) and Daniel Dennett, as well as the emergence of a new view of human functioning in the environment of new information and communication technologies. The aforementioned inter- 
disciplinary team of researchers as well as a number of other groups addressing the problems of relationships between brain work and the impact of the environment on the effects of this work, have influenced a new perception of the role and place of cyberspace in the educational process. The multidimensional nature of these considerations was also influenced by the vision of the society of network, created by Emanuel Castells (1996/2000), and theories created within the reflections on the society of knowledge. These discussions have had a significant impact on theories of education addressing the problems of using cyberspace in education.

The dynamically developing technological progress in the information and communication space has significantly accelerated the work on introducing network tools into the educational process. Mobile phones, smartphones and iPads have become a symbol of changes in people's communication. Universal access to cyberspace means that the aforementioned communication tools between people beyond the transmission of information have become a kind of participant in the communication game with a growing social role. We are dealing with a paradox because, on the one hand, man, who is a social being, wants to contact people and willingly uses technology to communicate, but at the same time information tools deepen his loneliness. The plasticity of our brain makes us able to adapt to the changes occurring in communication, we correct our actions in a changing environment.

New technologies have created unlimited communication possibilities in cyberspace, among other things, it allows for talking to a large number of people on any topic. Exchange of thoughts significantly changes the learning environment, causes various turbulences and causes a growing dissonance of educational expectations in relation to those offered by educational institutions.

Curiosity, which is a natural biological feature of human beings, has been strengthened with the tools of cyberspace that enable not only its full satisfaction but also open new possibilities of obtaining information. It evokes a constant pursuit of new information and pushes to the background reflections on the place of man in the world. In such an environment, there is no room for the development of interpersonal intelligence, defined by Howard Gardner.

\section{Cyberspace and virtual reality - general view}

In the didactic discourse we can meet at least four terms defining the concept of cyberspace. It is assumed to be a space for open communication via the Internet or other networks, which creates a system of information connections that facilitate 
contacts for users, including real time. Cyberspace is treated here as a kind of communication channel used to exchange information. The second term of this concept includes an indication of the area of graphics functioning in personal computers, and the third sets the sign of equality between cyberspace and virtual reality. At the same time, interchangeability of these two terms is accepted with all its consequences. In turn, the fourth approach requires seeing cyberspace in the dimension of art. It is assumed that this is an environment creating conditions for the cooperation of various media in order to create a work of art. This is a comprehensive set of data for creation of potential products of art.

Each of the presented approaches emphasizes another area of activity. From the perspective of cognitive pedagogy, what is the most appropriate is to recognize cyberspace in terms of electronic communication, functioning as a result of links in Internet networks. The characteristic features of this space are: information processing, computational character, occurrence of hypertext, plasticity, fluidity, and interactivity (P. Lévy, 1999, p. 32).

Cyberspace is treated as a communication environment enabling the use of research results carried out in the field of media pedagogy as well as theoretical achievements regarding communication models. Such an approach promotes transparency of the perception of social information exchange mechanisms occurring in the relations between the participants in the virtual conversation. It also allows for understanding their interaction using media language.

The concept of virtual reality in the literature of the subject has two basic references. In a narrower sense, the emphasis was placed on the technological side of the concept, which is close to the meaning of broadcast cyberspace. Virtual reality is defined here as the recipient's full sensory immersion in a virtual world generated by the information technology created by multimedia. They are characterized by an idealized, realistic world that allows for watching, feeling, acting and behaving as it does in the real world.

The wider definition of virtual reality implies the occurrence of experience resulting from contact with an artificial environment created by sensory stimuli. The activities of a person functioning in this environment are determined by what this environment brings. Therefore, man creates and participates in a world that has both a reference to imaginary and existing reality. Adoption of a wider view of virtual reality makes it possible to analyse the behaviour of each participant immersed in this space in terms of cognitive pedagogy.

The creator of the concept of virtual reality, Jaron Zepel Lanier, in his pioneering visions of the application of avatars in medicine, created the basis for the digital space of using IT tools in many areas of cyberspace. Modelled on the idea of 
Lanier Second Life, the development of IT tools that create the world of delusion was initiated. It is of two-way character due to the existing relationship between real and virtual life. For example, a "resident" of Second Life creates an artificial environment and at the same time participates in its "life". A participant in the virtual world acquires a specific experience that he/she uses in reality. In this digital space, universally appearing multimedia leaves deep traces in the human consciousness, which influence decisions regarding actions taken in everyday life. Thanks to this space, an alternative world is built, which affects the view of reality. The image of artificial reality created with the help of IT tools contains both simulations of the real world and fictional situations being the product of the imagination of its creators. This fact is essential for the psychological experiences generated by the recipient, which involve the multisensory state that arises in the communication process.

According to Patricia M. Greefield (2009, pp. 69-71), the use of cyberspace leads to widespread and sophisticated development of visual and spatial skills, but also weakens the ability to deeply process information. Meanwhile, this ability is the basis for acquiring knowledge with understanding, critical thinking as well as expanding imagination and reflection.

The definitions of cyberspace and virtual reality adopted for further analysis require analysing the communicative electronic space with the relations appearing in it and looking at the mechanisms contributing to the conscious creation of a vision of the world based on the imagination and the illusion produced.

We have known for a long time that man assimilates the culture in which he grows from birth. Initially, the range covers the immediate surroundings, and as he grows he systematically extends them. If there is cyberspace and virtual reality in the child's environment from birth, the acquired information will shape his/ her view of reality in a significant way. This overview will be strengthened by direct participation in the life of a given environment. Experiments conducted at the Torun Centre for Media Research indicate that the child's active involvement in virtual reality is more conducive to learning than traditional education. There must, however, be an important condition here, namely, the teacher's actions must be carefully planned and precisely implemented in the form of purposeful didactic undertakings. It was also observed that learners can obtain different results, which is related to the previous experience acquired while working in the network. A child working in virtual reality becomes independent and can easily find information. This has an impact on developing in the brain many tools for thinking (D.C. Dennett, 2013). The observations made at the research centre in Torun coincide with the studies conducted by Gary Small and Gigi Vorgan (2008), 
who noticed that the neuronal activity of people searching with the help of Google is different from that of those who did not use this IT tool.

Virtual reality is a powerful tool for emotional interaction, which affects the changes taking place in the human brain. This is particularly evident in children who are systematically operating in the virtual world. This translates into their way of thinking, solving problems, etc. Emotions are also necessary, provided we use them optimally in the learning process. This means that they cannot be too big or too small. Finding the right point of their intensity is very difficult, due to the individual threshold of optimality. Finding it requires a lot of experience and teaching skills from the teacher.

It is also worth mentioning that communication without emotions does not exist, they accompany all human activities also in cyberspace, where the media language (sound, image, animation) favours their production. A significant impact here is the awakening of the impression of reality and closeness in the recipient.

Emotions also serve the person to influence the environment, which also happens in cyberspace. Most often, we observe use of emotions to modify behaviours in order to obtain certain benefits. The use of emotions takes place in the social, political, economic and cultural dimensions, because it gives good results. Emotions are also used for manipulation of individuals and social groups. Understanding the mechanisms of causing emotional states is also important in achieving educational goals. It promotes an increase in the ability to acquire knowledge and skills.

\section{Cyberspace, virtual reality and learning}

Work in cyberspace is characterized by cyclic activity of the brain in the concentration-distraction of the attention system. Nicholas Carr (2010, p. 148) put it in the following way: "... the network focuses our attention only to distract it immediately". The mechanism of this phenomenon is similar to the one observed in the learning process with the use of television. We concentrate on the medium screen and at the same time the generated message distracts us (B. Siemieniecki, 1991). The speed of occurring changes results in the limitation of information flow and attention disorder. A similar situation occurs in cyberspace, where while designing the learning process we must take into account the presence of fluctuations in the concentration of the internet user. During this process, there is competition of various types of cultural information, known as memes (B. Siemieniecki, 2012). They have a significant impact on learning, so further consideration should focus on 
the factors that determine the efficiency of information transmission to the brain. By analysing the reception of information in cyberspace and in virtual reality, we can identify a number of common phenomena that are important for learning. These include: multitasking, cognitive load, sequencing mechanism, compression, and addiction.

In the everyday discourse on learning, the assumption is made that the brain can simultaneously perform many tasks in cyberspace. This is erroneous reasoning because in the case of simultaneous accomplishment of two tasks (listening to the text and simultaneous making of figures in the mind), the brain activity decreases by about $1 / 3$ in relation to the independent performance of both tasks (B. Siemieniecki, 2012a). The complexity of the task is a factor that significantly affects the efficiency of the activities performed. With simple activities, the delay may not occur or be small, but when complex, there is a greater involvement of the brain, and as a result, the efficiency of the activity decreases. This is caused by the distraction of attention

Research carried out at the Department of Education and Media in the Nicolaus Copernicus University in Torun has shown that in the case of memory solving of a mathematical task by students who did not watch a cartoon on TV and those who watched it there was a time difference. The students needed almost twice as much time to perform a mathematical task as the viewing and counting activities were performed simultaneously. The aforementioned Carr (2010, pp. 165-166) believes that this is due to the so-called costs of switching attention, resulting from the need for the reorientation of the brain. Therefore, the use of cyberspace to achieve learning goals involving the acquisition of new skills requires didactic activities that limit the distraction of attention because reflection on the material to acquire requires concentration.

During the research at the Department of Education and Media in Torun, another phenomenon was also observed: the more automatically performed activity related to the computer program, the more efficiently the proper mathematical task was made, even if it was complicated. The observations cited, resulting from the conducted research, indicate that the exercise of operating schemes increases the results in the field of the automation of given activities, but multitasking is not conducive to the learner's proper assessment, synthesis and conclusion.

According to Anders Ericsson and Robert Pool (2016), acquiring knowledge is not determined by inborn talents and specific predispositions, but by the way of learning. It is connected with the continuous development of a person. Therefore, if we do not want to stay in one place, we have to cross borders and not stop at mastering automated activities. This requires continuous transgressive 
action, which Jerome Bruner signalled many years ago. As a result, if we want to succeed in learning using cyberspace for this purpose, we must apply the proper way of acquiring knowledge and make enough effort. Experiments conducted at the Centre for Media Research in Torun indicate that the use of cyberspace in education promotes the acceleration of learning, but requires diligence in designing didactic activities. This applies to both improvement and acquisition of new skills. An important role is also played by a multilevel and individualized learning system that takes into account both gifted students and those with fewer biological capabilities.

In the era of smartphones, multitasking becomes an important adjustment factor to the requirements of the social environment. The introduction of an electronic tablet with access to cyberspace instead of a notebook is not only a change of the tool, but it is first of all a new socio-cultural environment, creating new opportunities, and new didactic problems, e.g., related to the change of the hierarchy of priorities. A learner surrounded by many contexts created by virtuality is confronted with the choice of information and taking on new challenges. This requires not only proficiency in using IT tools but also brain training to improve attention. An additional difficulty is the interpenetration of mutual problems occurring in cyberspace and reality. As a result, students have a number of barriers that often prevent achieving a goal, which is why the school is becoming an important centre of preparation for coping in life and in cyberspace. In addition to training, it is also necessary to conduct therapeutic activities, limiting the occurrence of difficulties encountered by participants in the educational process.

Increasing the amount of information processed by students results in cognitive load. It was described in detail by John Sweller (1998), who studied, for many years, how the human mind processes its information resources during learning. His theory assumes that learning proceeds best when it is compatible with cognitive activity and when the cognitive load associated with solving the task is reduced.

Sweller claims that the multimedia message is less distorting short-term memory and allows it to increase its capacity. However, there must be an important condition for the information transmitted by different channels (visual, auditory) to complement each other. If this is not the case, an additional burden arises because information items compete with each other causing distraction.

Stopping the information reaching the brain is connected with the functioning of the sequencing mechanism. It consists in postponing the reaction during the maximum overload with information and its storage in the temporary memory store. This process releases the brain's executive activities and is done automatically. 
An important area of analysis in cognitive pedagogy are issues related to addiction. The problem of addictions in cyberspace is of particular importance due to its universal accessibility through digital communication tools. The mechanism acting in the brain, roughly speaking, is similar to that of drug or alcohol addiction. Neurons forming the "reward centre" stimulate activities aimed at connecting to cyberspace and undertaking activities related to searching for information. Reward in the form of pleasure encourages further action, resulting in the creation of a specific mechanism of addiction.

When we talk about addiction in cyberspace, we mean the applications in it that our brain is in contact with. It is a broad spectrum of various tools and related activities that can help us lose control over what should be the norm. This applies, e.g., to online shopping, continuous search of websites for specific information, or participation in network gambling. Because the strength of addiction is related to individual biological predispositions, there is a significant difference in the diagnosis, which makes it difficult to detect and counter this destructive phenomenon at an early stage. The fight against cyberspace addiction is also difficult because our brain is designed to search for information and to boost activities aimed at cooperation with the media. A good example is the use of smartphones. We will not immediately notice the effects of intensive use of this medium because many activities take place in the area of unconscious space and the actions taken often seem to be a natural process of seeking information. The operations that take place in the unconscious space, which are of incubative nature, have long been known to didactics investigating the process of solving problems. Activity in cyberspace contributes to the construction of new neuronal circuits in the brain, which are then used in many other thinking processes. Nicolas Carr points out that searching websites also has a positive aspect because it involves many brain functions that improve the functioning of this organ. Therefore, it can be helpful in maintaining the mental capacity of older people.

When working in cyberspace, the excess of information flowing into the brain in connection with time pressure causes a reaction in the form of limitation of intellectual activities. This in turn results in the superficiality of the analyses being carried out, as well as leaving the area in the network and abandoning the solved task. This phenomenon is observed, among other things, during experiments requiring a deeper analysis of information and reflection on the selection of one correct variant among many.

Another important problem in cognitive pedagogy is the functioning of language in cyberspace. Because it is of considerable importance for intellectual development, it should be the subject of a broader debate. Until now, the focus 
has been primarily on external attributes, e.g., the choice and type of words used, the formulation of thoughts, etc. Meanwhile, it is more important to determine the relationship between the language used and the development of the neural network. Although studies on the deciphering of human thought are conducted very intensively, it is more important for education to indicate the relationships occurring in the teaching - learning process. The first such attempts were made, among others, at the Brain Mind Institute in the Swiss Ecole Polytechnique Federale de Lausanne (EPFL). They include experiments in which the possibilities of directing a stream of information to the brain via the computer interface are studied. The director of the said research centre, Henry Markram, believes that an educational program designed to allow students to acquire certain skills in the shortest possible time can be created (G. Stix, 2008). The time factor is a central element in the search for new didactic solutions because two basic tasks are faced by modern education: effective education and its implementation as soon as possible.

Analysing cyberspace from the perspective of cognitive pedagogy, besides communication and information, there is another area important for education - the language of the media. There have been many publications on this subject, which is why an important problem has been addressed in this article, i.e., the relationship between language and expanding the possibilities of the mind.

In his arguments, Michael Tomasello (1999) draws attention to the relationship between the use of natural language and shaping the nature of human cognition. According to the aforementioned author, mastering language symbols leads to the development of completely new cognitive representations. Adoption of this thesis has far-reaching consequences for cyberspace and virtual reality. The mind of man operating in a digital world dominated by the media language extends our capabilities. The IT tools used leave a lasting impression in our mind (B. Siemieniecki 2010). The language of the media, thus, influences the social evolution of Homo sapiens and, in the long run, the course of events in the course of biological evolution. There are also poorly researched phenomena that do not always serve only the good of man.

\section{Conclusion}

The presented brief picture of phenomena occurring in cyberspace seen from the perspective of cognitive pedagogy indicates the need for a new look at education. The complexity of existing problems in the communication and information space requires the extension of didactic analyses for the processing of information 
in the human brain. Studies carried out so far indicate that education faces a great challenge because there is a need for a new interdisciplinary approach to the phenomena occurring in cyberspace. Cognitive pedagogy must also undertake research on the behaviour of virtual reality participants, where processes permanently changing their brains occur. The combination of the real and digital world is not only a change in communication but, above all, a new culture of social relations and a new language combining the one used so far by people with a new media language. The problems highlighted in the article constitute a general outline of a large area of research, which must be the subject of the search for modern education.

\section{References}

Brockman J., (1995) The third culture. Beyond the scientific revolution, Rockefeller Centre, New York.

Car N., The Shallows. (2010) What the Internet Is Doing to Our Brains, W.W. Norton \& Company Inc. London.

Castells E., (1996/2000) The rise of the network society, Blackwell, Oxford.

Dennett D.C., (2013) Intuition Pumps and Other Tools for Thinking. W.W. Norton Company.

Ericsson A., Pool R., (2016) Peak: Secrets from the New Science of Expertise, April $5^{\text {th }}$ 2016 by Eamon Dolan/Houghton Mifflin Harcourt, Kindle Edition, Boston-New York. Greenfield P.M., (2009) Technology and Informal Education. What Is Taught, What Is Learned, Science, nr 323, s. 69-71.

Lévy P., (1999) Collective intelligence: mankind's emerging world in cyberspace Perseus Books, Cambridge, Massachusetts.

Small G., Vorgan G., (2008) iBrain. Surviving the Technological Alteration of the Modern Mind, William Morrow.

Siemieniecki B., (1991) Środki dydaktyczne w procesie myślenia twórczego, Prace Monograficzne Wyższej Szkoły Pedagogicznej w Krakowie, T. 134, Wydawnictwo Naukowe WSP, Kraków, (B. Siemieniecki, (1991) Teaching aids in the process of creative thinking, Monographic works of the College of Pedagogy in Cracow, T. 134, WSP Scientific Publishers, Cracow).

Siemieniecki B., (2012) Introduzione alla pedagogia cognitiva, Armando Editore (January $1,2012)$ Italy.

Siemieniecki B., (2012a) Biologiczne mechanizmy odbioru informacji a pedagogika. Kognitywistyka i Media w Edukacji nr 1, pp. 88-97 [Biological mechanisms of information reception and pedagogy. Cognitive Science and Media in Education, No. 1, pp. 88-97].

Siemieniecki B., (2010) Kognitive sprakproblemer, In: D. Siemieniecka, B. Siemieniecki, H. Nilsen, E. Bratland, Sprak i medier, Wydaw. Naukowe UMK, Toruń. 
Stix G., (2008) Jacking into the Brain - Is the Brain the Ultimate Computer Interface? Scientific American, November, Volume 299, Issue 5, 56-61.

Sweller, J., Van Merrienboer, J.J., \& Paas, F.G., (1998) Cognitive architecture and instructional design. Educational psychology review, 10 (3), pp. 251-296.

Tomasello M., (1999) The Cultural Origins of Human Cognition. Harvard University Press, Cambridge, Massachusetts, London, England. 